\title{
RESEARCH
}

Open Access

\section{Loss-of-function mutations in KEAP1 drive lung cancer progression via KEAP1/NRF2 pathway activation}

Meiling Gong ${ }^{1 \dagger}$, Yan $\mathrm{Li}^{2,3+}$, Xiaoping Ye ${ }^{2}$, Linlin Zhang ${ }^{4}$, Zhifang Wang ${ }^{1}$, Xiaowen $\mathrm{Xu}^{1}$, Yejing Shen ${ }^{1}$ and Cuixia Zheng $^{1 *}$

\begin{abstract}
Background and purpose: Targeted therapy and immunotherapy have led to dramatic change in the treatment of lung cancer, however, the overall 5-year survival rate of lung cancer patients is still suboptimal. It is important to exploit new potential of molecularly targeted therapies. High-frequency somatic mutations in KEAP1/NRF2 (27.9\%) have been identified in lung squamous cell carcinoma. In this research, we explored the role of KEAP1 somatic mutations in the development of LSCC and whether a nuclear factor erythroid 2-related factor 2(NRF2) inhibitor be potential to target lung cancer carrying KEAP1/NRF2 mutations.

Methods: Lung cancer cell lines A549 and H460 with loss-of-function mutations in KEAP1 stably transfected with wild-type (WT) KEAP1 or somatic mutations in KEAP1 were used to investigate the functions of somatic mutations in KEAP1. Flow cytometry, plate clone formation experiments, and scratch tests were used to examine reactive oxygen species, proliferation, and migration of these cell lines.

Results: The expression of NRF2 and its target genes increased, and tumor cell proliferation, migration, and tumor growth were accelerated in A549 and H460 cells stably transfected with KEAP1 mutants compared to control cells with a loss-of-function KEAP1 mutation and stably transfected with WT KEAP1 in both in vitro and in vivo studies. The proliferation of A549 cell line trasfected with the R320Q KEAP1 mutant was inhibited more apparent than that of the A549 cell line trasfected with WT KEAP1 after treatment with NRF2 inhibitor ML385.

Conclusion: Somatic mutations of KEAP1 identified from patients with LSCC likely promote tumorigenesis mediated by activation of the KEAP1/NRF2 antioxidant stress response pathway. NRF2 inhibition with ML385 could inhibit the proliferation of tumor cells with KEAP1 mutation.
\end{abstract}

Keywords: KEAP1/NRF2, Somatic mutation, NRF2 inhibitor, Lung carcinoma, Targeted therapy

\footnotetext{
* Correspondence: zcx9566@163.com

${ }^{\dagger}$ Meiling Gong and Yan Li contributed equally to this work.

'Department of Respiratory and Critical Care Medicine, Yangpu Hospital,

Tongji Universtiy School Of Medicine, Shanghai, China

Full list of author information is available at the end of the article
}

C C The Author(s). 2020 Open Access This article is licensed under a Creative Commons Attribution 4.0 International License, which permits use, sharing, adaptation, distribution and reproduction in any medium or format, as long as you give appropriate credit to the original author(s) and the source, provide a link to the Creative Commons licence, and indicate if changes were made. The images or other third party material in this article are included in the article's Creative Commons licence, unless indicated otherwise in a credit line to the material. If material is not included in the article's Creative Commons licence and your intended use is not permitted by statutory regulation or exceeds the permitted use, you will need to obtain permission directly from the copyright holder. To view a copy of this licence, visit http://creativecommons.org/licenses/by/4.0/. The Creative Commons Public Domain Dedication waiver (http://creativecommons.org/publicdomain/zero/1.0/) applies to the data made available in this article, unless otherwise stated in a credit line to the data. 


\section{Background}

Lung cancer is a leading cause of cancer-related death, with a 5-year overall survival rate less than $15 \%$ [1], a significantly lower survival rate than that of most epithelial malignancies. Lung cancer is divided into small cell lung cancer and non-small cell lung cancer (NSCLC). The proportion of the NSCLC type is more than 85\%, mainly including lung adenocarcinoma and lung squamous cell carcinoma $[2,3]$. Lung squamous cell carcinoma (LSCC) accounts for approximately $30 \%$ of all lung cancers, with a extremely high mortality rate [4]. Because most lung cancer patients are already in the advanced stage of disease at the time of diagnosis, they have lost the opportunity for surgical treatment, and the prognosis of LSCC has not obviously improved, although the finding of high-frequency mutations in epidermal growth factor receptor (EGFR) kinase has led to a dramatic change in the treatment of patients with lung adenocarcinoma $[5,6]$, and recent data have indicated that targeting mutations in $B R A F$, $A K T 1, E R B B 2$, and PIK3CA as well as fusions that involve receptor tyrosine kinase genes $A L K, R O S 1$, and $R E T$ may also be successful $[7,8]$. Unfortunately, the activating mutations in EGFR and $A L K$ fusions are limited in lung adenocarcinoma and are not present in LSCC [9], and targeted agents developed for these activating mutations are largely ineffective in LSCC.

Recent researches have accumulated approximately 29 possible pathogenic genes for LSCC and are widely accepted [10-12]. However, therapeutic drugs targeting these driver genes are lacking. Interestingly, a search of the TCGA database revealed that approximately $30 \%$ of LSCCs undergo recurrent mutations in KEAP1 and NFE2L2(also named as NRF2) [11, 12]. In our previous study, we identified that KEAP1 and NRF2 mutations are recurrent in Chinese patients with LSCC, with a $5.8 \%$ frequency for $K E A P 1$ and a $27.9 \%$ frequency for KEAP1/NRF2 mutations. However, mutations in KEAP1/NRF2 in Chinese patients with lung adenocarcinoma are rarely found, which is consistent with reports from Takahashi $\mathrm{T}$ [13]. Interestingly, KEAP1 and NRF2 mutations show mutual exclusive in Chinese patients with LSCC [12]. KEAP1 and NRF2 are the two key genes that regulate the oxidative stress pathway. At physiological homeostasis, NRF2 is bound by the adapter protein KEAP1, which recruits the CUL3 ubiquitin ligase, leading to the proteasomal degradation of NRF2 [14]. Oxidative stress acts on KEAP1, causing its conformation change and dissociation from NRF2, thereby losing the ability to mediate NRF2 degradation $[15,16]$ and leading to NRF2 activation and subsequent antioxidative properties, which is important in maintaining physiological homeostasis. However, it has been reported that NRF2 activation involves in chemotherapy drugs inactivation through rapid metabolism of these drugs in cells, significantly reducing their anti-tumor efficacy [17-19]. More recently, the data have also shown that loss of function of KEAP1 promotes KRAS-driven lung cancer and results in the dependence on glutaminolysis [20].

Therefore, we aimed to test whether mutations in $K E A P 1$, identified in our previous study, accelerate the development of lung cancer, and whether a NRF2 inhibitor can be used as a targeted therapeutic drug in patients with lung cancer carrying KEAP1/NRF2 mutations.

\section{Materials and methods}

\section{Cell culture, reagents, and nude mice}

The NCI-H1299,A549, H838, H460,H1299, 95D, and SPCA1 human lung cancer cell lines and HEK293T cells were obtained from American Type Culture Collection (Manassas, VA, USA). H1299, H838, H460, H292, 95D, and SPCA1 cells were maintained in RPMI 1640 medium (Gibco, Grand Island, NY, USA). A549 cells were cultured in F-12 K(Gibco) supplemented with $10 \%$ fetal bovine serum (Gibco) at $37^{\circ} \mathrm{C}$ in a humidified atmosphere containing 5\% CO2. Twelve 4-6-week-old male $\mathrm{BALB} / \mathrm{c}$ nude mice were purchased and reared from the Shanghai Ninth People's Hospital Central Laboratory Animal Law.

\section{Plasmids, site-directed mutagenesis, and stable transfection}

Mutations were conducted using the QuikChange sitedirected mutagenesis kit (Stratagene, La Jolla, CA, USA) and were validated by sequencing; the primer sequences for mutagenesis are shown in (Supplementary Table 1). A retrovirus-mediated infection system was used to construct A549 and H460 cells stably over-expressing 3FLAG-tagged KEAP1(WT or mutant). For PMSCV production, DNA encoding 3FLAG-tagged KEAP1 was inserted into the multi-cloning site of the $\mathrm{pMSCV}$ vector. Each PMSCV vector was co-transfected with gag-pol and VSVG using Lipofectamine 2000(Invitrogen,Waltham, MA, USA) in $293 \mathrm{~T}$ cells. The virus was collected 2 days later and was transfected into A549 and H460 cells. The infected cells were selected with $1 \mu \mathrm{g} / \mathrm{mL}$ (A549) or $0.5 \mu \mathrm{g} / \mathrm{mL}$ (H460) of puromycin for $3-4$ weeks.

\section{Gene editing using CRISPR/Cas9 system}

Target-specific guide RNA within NRF2 gene locus was designed on CRISPRDESIGN (http://crispr.mit.edu/). The following target sgRNA sequences were used in this study:sgRNA-F 5'-TGCCTGTAAGTCCTGGTCAT-3', sgRNA-R 5' -TCTCTGGTGTGTTCTCACAT-3'. Igonucleotides for guide RNA were inserted into CRISPR Nuclease vector and then the vector was transfected into 
A549 cells using Lipofectamine 2000 (Invitrogen) according to the manufacturer's instructions. Specific lentivirus transfecting method was the same as above. Finally, 3 homozygous knockout cell lines were selected (Supplementary Fig. 1a).

\section{Western blot and immunoprecipitation analyses}

The antibodies used in our study were as follows: antiFLAG M2 monoclonal (Sigma, St. Louis, MO, USA), anti-NRF2 (Abcam, Cambridge, UnitedKingdom), anti$\beta$-actin (Cell Signaling,Danvers, MA, USA), anti-HO-1 (Cell Signaling), anti-LaminB1 (Cell Signaling), antiLamin A/C (Cell Signaling). The Nuclear and Cytoplasmic Protein Extraction kit was obtained from (Thermo Fisher Scientific, Waltham, MA, USA). For immunoprecipitation, Whole cell lysate (WCL) was used respectively as the negative control. Cell lysates were cleared by centrifugation and were incubated with FLAG resin (Sigma) before washing with lysis buffer, followed by overnight incubation at $4{ }^{\circ} \mathrm{C}$. After washing three times by $1 \times$ phosphate-buffered saline (PBS), the precipitates were analyzed by immunoblotting.

\section{Real-time quantitative PCR}

Total RNA was prepared from cells using Trizol reagent (Invitrogen), and tumors using Animal Total RNA Isolation kit (Sangon Biotech,Shanghai, China) and reverse transcription were performed using the PrimeScript RT reagent kit with gDNA Eraser (Takara Bio,Shiga, Japan). The sequences for each primer are listed in Supplementary Table 2.

\section{ROS measurement}

After the cells were washed with PBS twice, the cells were incubated with $1 \mu \mathrm{M}$ CM-H2DCF-DA (Nanjing Jiancheng, Nanjing, China) in culture conditions for 30 min in the dark and then were trypsinized and collected. The ROS levels were examined by flow cytometry.

\section{Colony-formation assay}

Exponentially growing cells were counted,diluted, and seeded in triplicate at 800-1000 cells/well in 6-well plates. To assess clonogenic survival following drug exposure, the cell cultures were incubated in complete growth medium at $37^{\circ} \mathrm{C}$ for $11-14$ days. Colonies were fixed with precooled methanol and then were stained with $0.5 \%(\mathrm{w} / \mathrm{v})$ crystal violet for $30 \mathrm{~min}$ at room temperature, followed by washing with PBS, photographing and counting. Only colonies with more than 50 cells were counted [21].

\section{Wound-healing assay}

Cell motility was determined by measuring the movement of cells close to an artificial wound. Cells were wounded with a $200-\mu \mathrm{L}$ pipette tip, washed with PBS, and incubated in F12-K or RPMI 1640 medium without FBS. The distances removed by cells were monitored by microscopy at the indicated time points. The scratched area was analyzed using ImageJ.

\section{Tumor xenograft model}

Twelve BALB/c nude mice (4-6 weeks old, male) were randomly assigned to two groups (WT or mutant). We infected A549-KEAP1-WT or A549-KEAP1-R320W cells $\left(1 \times 10^{8}\right)$ subcutaneously into the right flank of BALB/C nude mice and measured the tumor dimensions by calipers every 3-4 days. The tumor volumes were calculated using the formulalength $\left[(\mathrm{mm}) \times\right.$ width $\left.(\mathrm{mm})^{2}\right] / 2$ [22] All experimental protocols conducted on mice were performed in accordance with the National Institutes of Health (NIH) guidelines and were approved by the Shanghai Jiaotong University Animal Care and Use Committee.

\section{Statistical analysis}

All experiments were performed in quadruplicate and were repeated at least three times with similar results unless otherwise indicated. All statistical analyses were performed using unpaired two-tailed Student's t-test and the mean \pm standard error of the mean. A $P$-value of 0.05 or less was considered statistically significant. These analyses were performed using SPSS 13.0 software (SPSS Inc., Chicago, IL, USA) or GraphpadPrism 7 (GraphpadSoftware, San Diego, CA, USA).

\section{Results}

The KEAP1/NRF2 pathway was activated in lung cancer cell lines with KEAP1 mutations

To explore the effect of KEAP1 loss on activation of the KEAP1/NRF2 pathway, we collected three NSCLC cell lines with KEAP1 mutations (A549, NCI-H460, and NCI-H838) and four NSCLC cell lines without KEAP1/ NRF2 mutations (NCI-H1299, NCI-H292, 95D, and SPCA1). In the present study, we have found the frequency of KEAP1/NRF2 mutation was higher in human LSCC than that in LUAD patients, thus we hope find the LSCC cell lines with KEAP1 mutation to investigate the effect of KEAP1 mutation on the function of KEAP1/NRF2 pathaway. However, after serching the literatures and cell line database, we found none LSCC cell lines carried KEAP1/NRF2 mutation. We further collected all available 7 lung cancer cell lines and found three of them carried the KEAP1 mutation (A549, NCIH460, NCI-H838), thus, in the present study, all the seven lung cancer cell lines were used to study the influence of KEAP1 mutation on the function of KEAP1/ $N R F 2$ pathway. As expected, Sanger sequencing revealed that the A549, H460, and H838 cell lines carried 
A

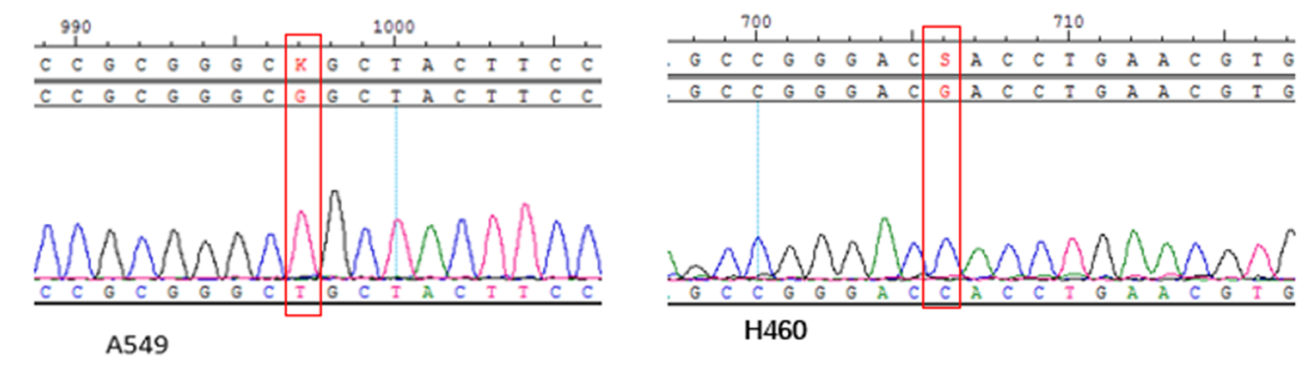

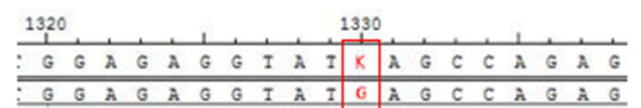

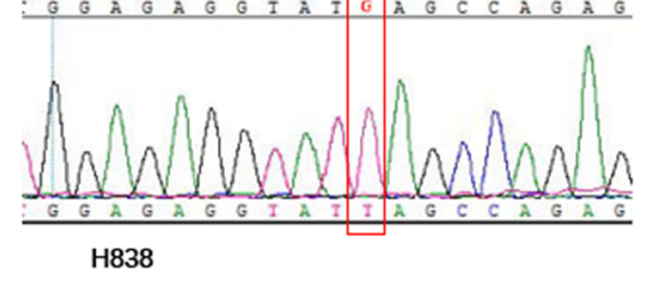

B
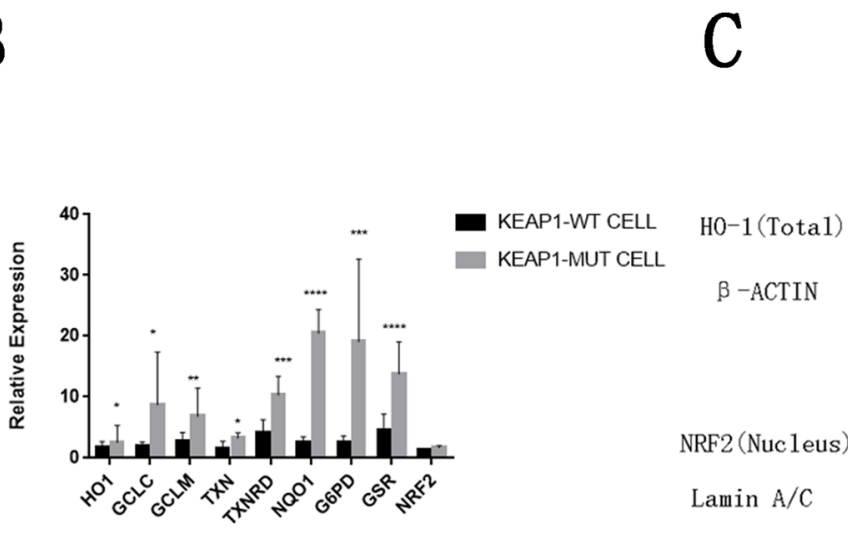

NRF2 (Nucleus)

Lamin $\mathrm{A} / \mathrm{C}$
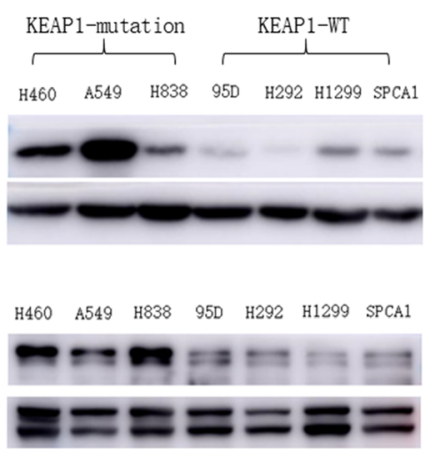

D
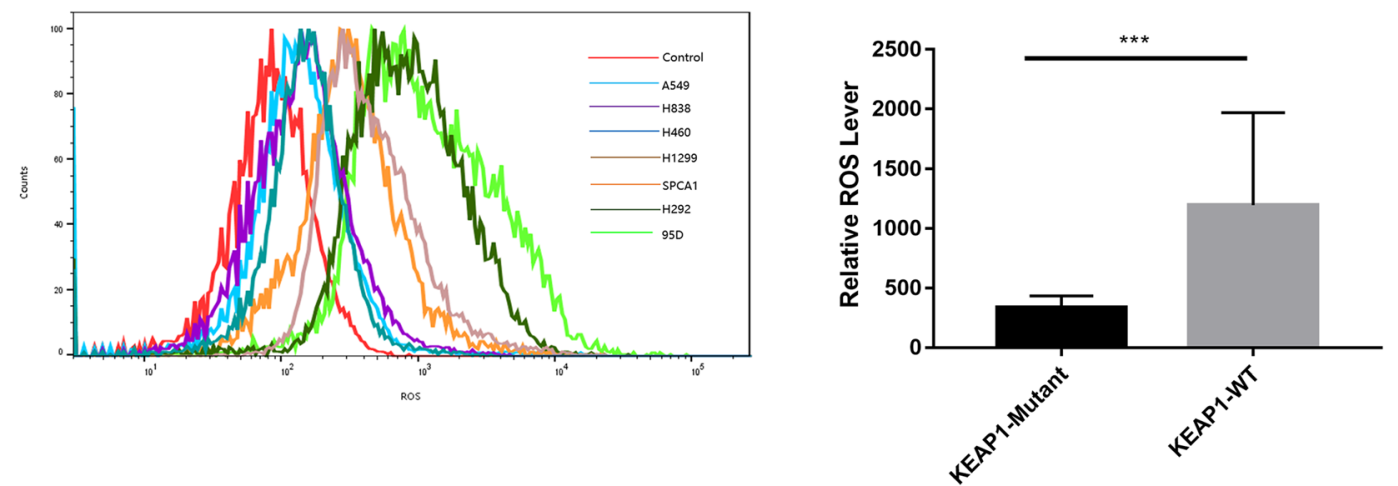

Fig. 1 (See legend on next page.) 
(See figure on previous page.)

Fig. 1 The KEAP1/NRF2 pathway was activated in lung cancer cell lines with loss-of-function mutations in KEAP1. a The mutations of KEAP1 in A549, H460, and H838 cells were detected by Sanger sequencing. The red rectangle indicates the mutation sites of KEAP1. b Compared with lung cancer cells with neither KEAP1 nor NRF2 mutation, the expression levels of NRF2 target genes were significantly increased in lung cancer cells with KEAP1 mutation. $\mathbf{c}$ The protein expression levels of NRF2 and its target protein HO-1 were increased in lung cancer cells with KEAP1 mutation compared with those in lung cancer cells with neither KEAP1 nor NRF2 mutation. $\mathbf{d}$ KEAP1 mutation suppressed ROS production in lung cancer cells. The fluorescence intensity in cells was detected by flow cytometry. Quantitative analysis of the mean fluorescence intensity using unpaired two-tailed Student's t-test. The results are expressed as mean \pm standard error of the mean. ${ }^{*} P<0.05,{ }^{*} P<0.01,{ }^{* *} P<0.001$, and ***** $P<0.0001$

homozygous point mutations at D236H, G333C, and E444* in KEAP1, respectively; however, H1299, NCIH292, 95D, and SPCA1 cell lines did not carry mutations in neither KEAP1 nor NRF2(Fig. 1a). Next, we found that the mRNA expression of downstream target genes of the KEAP1/NRF2 pathway, such as GCLC, GCLM, TXN, TXNRD, HO1, NQO1, GSR, and G6PD, which encode detoxifying enzymes and antioxidant proteins, were significantly higher in KEAP1 mutant cell lines than in wild-type (WT) lung cancer cells by realtime polymerase chain reaction (PCR), but the mRNA expression of NRF2 showed no significant difference between lung cancer cell lines with and without KEAP1 mutation (Fig. 1b). Given that NRF2 protein translocates into the nucleus to activate the transcription of downstream target genesin the KEAP1/NRF2 pathway, we further examined the protein expression of nuclear NRF2 and the downstream target HO-1 in these tumor cells by western blot analysis. As expected, function loss of KEAP1 significantly increased nuclear NRF2 levels and HO-1 levels incytoplasm (Fig. 1c). Thus, KEAP1 loss decreased the degradation of NRF2 to activate the KEAP1/NRF2 pathway.

Activation of the KEAP1/NRF2 pathway decreases intracellular reactive oxygen species (ROS) levels through upregulating the expression of detoxifying enzymes and antioxidant genes. Thus, we checked whether KEAP1 loss could decrease ROS production in lung cancer cell lines. As shown in Fig. 1d, ROS levels were significantly decreased in lung cancer cells with KEAP1 mutation. Collectively, these findings demonstrate that KEAP1 loss can enhance the ability of lung cancer cells to resist oxidative stress.

\section{Mutations in KEAP1 identified in Chinese patients with lung cancer promoted tumorigenesis via activation of the KEAP1/NRF2 pathway in lung cancer cells}

In our previous study, we identified five nonsynonymous mutations in KEAP1 from five patients with LSCC. However, the role of these KEAP1 mutations in tumorigenesis is unclear. The function these five nonsynonymous mutations in KEAP1 were predicted by Polyhen2_HDIV and SIFT. The results showed that these five nonsynonymous mutations in KEAP1 were harmful, affecting protein function (Table 1).

To verify whether these five somatic mutations in $K E A P 1$ influence the function of KEAP1, WT and five KEAP1 mutants were stably transfected with retroviral vectors into A549/H460 lung cancer cell lines that carry loss-of-function mutations in KEAP1. As expected, nuclear NRF2 protein levels and expression of the NRF2 target gene HO-1 were significantly decreased after A549 or H460 lung cancer cell lines were stably transfected with WT KEAP1 (Fig. 2a). However, the levels of nuclear NRF2 protein and expression of NRF2 target gene $\mathrm{HO}-1$ showed no significant difference after A549 or H460 lung cancer cell lines were stably transfected with these five KEAP1 mutants (Fig. 2a). These lung cancer cell lines that carry loss-of-function mutations in KEAP1, were losing the ability to mediate NRF2 degradation. Activated NRF2 will be degradated when WT KEAP1 overexpression.

Compared with A549 or H460 lung cancer cell lines stably transfected with empty vector, mRNA levels of NRF2 and its target genes HO-1, GCLC, and FTH1 were significantly decreased after the cell lines were stably transfected with WT KEAP1 (Fig. 2b). However, mRNA levels of NRF2 and its target genes $H O-1, G C L C$, and FTH1 were significantly increased in the A549 or H460 lung cancer cell lines stably transfected with these five KEAP1 mutants (Fig. 2b). Together, these data suggested that these five nonsynonymous mutations in KEAP1, derived from Chinese patients with LSCC, were loss-offunction mutations that upregulated the expression of detoxifying enzymes and antioxidant genes.

KEAP1 is located at $19 \mathrm{p} 13.2$ and its protein has three major domains: an N-terminal broad complex, tramtrack, and the bric-a-brac (BTB) domain; a central intervening region (IVR); and a series of six C-terminal Kelch repeats [23]. The Kelch repeats of KEAP1 bind the NEH2 domain of NRF2 [24], whereas the IVR and BTB domains are required for the redox-sensitive regulation of NRF2 through a series of reactive cysteines present throughout this region $[14,25]$. In our present study, we found somatic mutations at R320Q, R413L, and D479H in the Kelch repeat domains of KEAP1, a somatic mutation at R234W in the IVR domain, and a somatic 


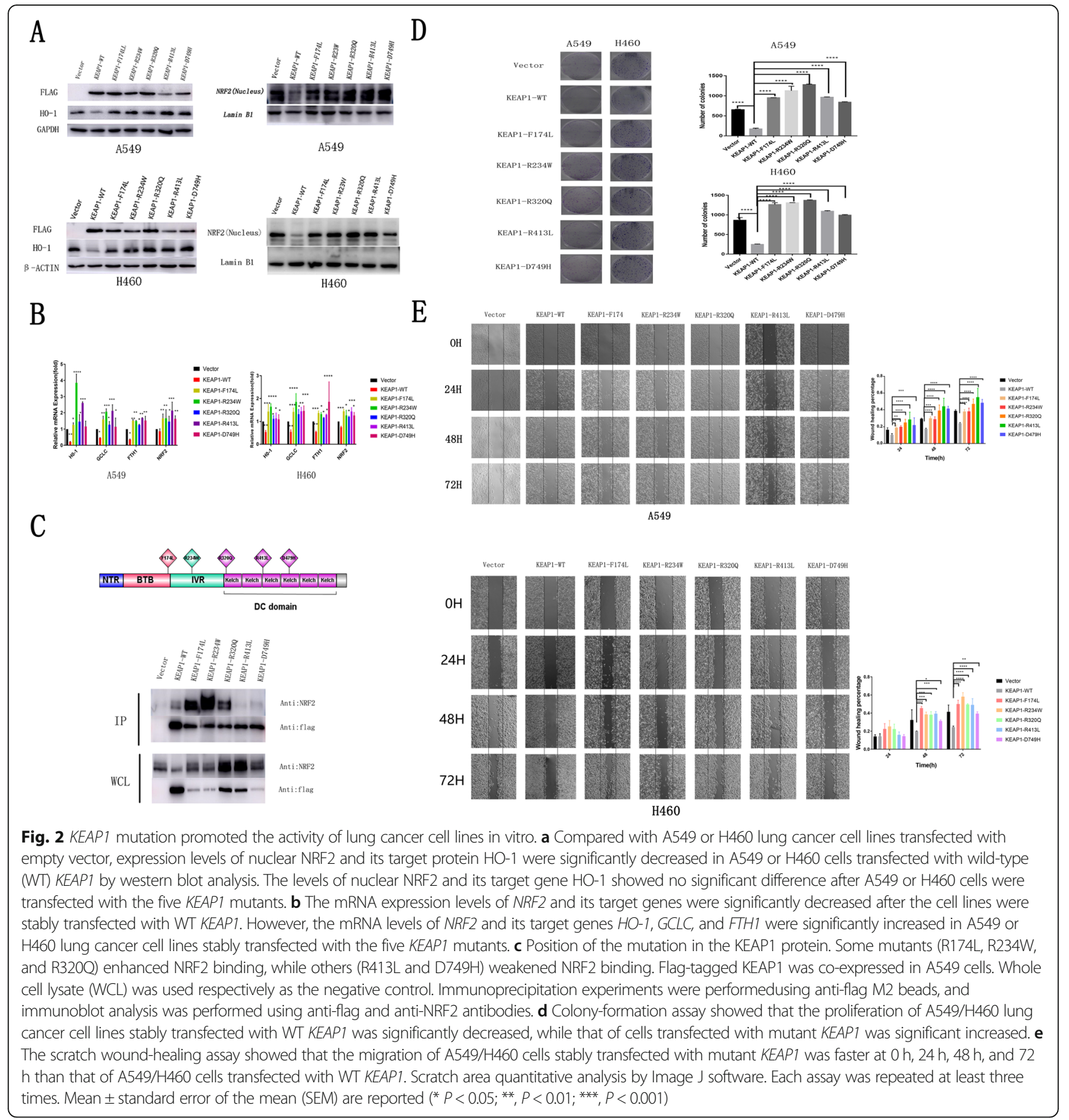

mutation at F174L in the BTB domain of KEAP1 (Fig. 2c). The binding of KEAP1 mutants to NRF2 was detected in coimmunoprecipitation experiments. Interestingly, mutants at $\mathrm{R} 413 \mathrm{~L}$ and $\mathrm{D} 479 \mathrm{H}$ in the Kelch repeat domain of KEAP1 did not bind to NRF2 (Fig. 2c). However, compared with WT KEAP1, binding of the mutants at F174L in the BTB domain and at R234W in the IVR domain of NRF2 was significantly increased (Fig. 2c). Unexpectedly, binding of NRF2 to the KEAP1 mutants at R320Q in the Kelch repeat domain was not affected (Fig. 2c).
To uncover whether these five somatic mutations in KEAP1 influence the biological behavior of lung cancer cells, cell proliferation and migration were detected by colony-formation and scratch experiments. Compared with A549/H460 lung cancer cell lines stably transfected with retroviral empty vector, the colony formation and migration of A549/H460 lung cancer cell lines stably transfected with WT KEAP1 were significantly decreased (Fig. 2d, e). However, after being stably transfected with KEAP1 mutants, the colony formation and migration of 
A549/H460 lung cancer cell lines significantly increased (Fig. 2d, e). To further validate that their mechanism were really passed through the NRF2 pathway, we knockdown NRF2 using double sgRNAs and detected the expression of well established downstream genes of NRF2. As shown in (supplementary Fig. 1b, c), the expression of NRF2 target gene HO-1protein level was significantly decreased and the mRNA level of HMOX1, HO-1, GCLC, NQO1, FTH1, NRF2 when NRF2 was knockdown. As expected (supplementary Fig. 1d, e), the reduced number of colonies and migration in cells knockouted with NRF2.These data suggest that the newly found somatic mutations in KEAP1 promote tumor cell activity through activating NRF2 antioxidant stress signaling pathways.

\section{The somatic mutation at $\mathrm{R} 320 \mathrm{Q}$ in KEAP1 accelerated tumor growth in vivo}

Due to we have considered that R320Q mutant has a considerable effection to KEAP1'S function, the ability of the antioxidative stress or proliferation and migration was significantly increased. Therefore, we choosed the
R320Q mutant for our next experiments. To further examine the effect of the somatic mutations in KEAP1 on the growth of lung cancer cell lines in vivo, the A549 cell lines stably transfected with WT KEAP1 or the R320Q mutant of KEAP1 were grafted subcutaneously into 4 to 5-week-old nude mice. After the cancer cell lines were grafted subcutaneously into nude mice, tumor sizes were measured using Vernier caliperseach day for 4 days. After 45 days of subcutaneous engraftment, tumors were peeled from the subcutis of the nude mice. The tumor sizes of the A549 cell line stably transfected with the R320Q KEAP1mutant were significantly larger than those of the A549 cell line stably transfected with WT KEAP1 (Fig. 3a). Additionally, the tumor growth of the A549 cell line stably transfected with the R320Q KEAP1 mutant was strongly accelerated compared with that of the A549 cell line stably transfected with WT $K E A P 1$, as measured by the change in tumor volume (Fig. 3b). Consistent with the in vitro results, the KEAP1 mutant showed significantly accelerated tumor growth in vivo. These results indicate that KEAP1 likely is a novel tumor driver gene for LSCC.

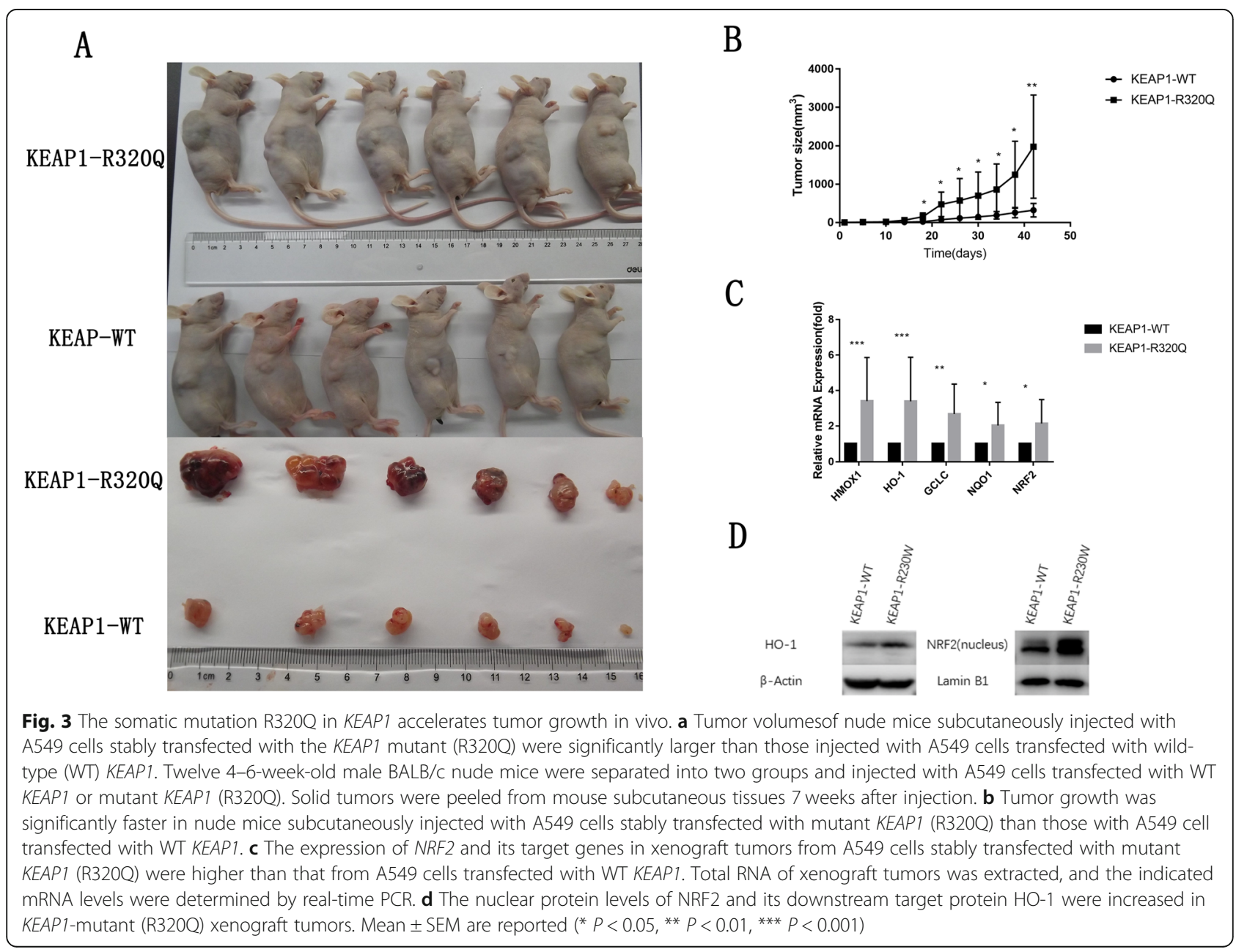


Next, we examined the expression of oxidative stressrelated genes in the grafted tumor tissues from the nude mice. Compared with the expression of NRF2 in the nucleus and its target protein $\mathrm{HO}-1$ in the cytoplasm in tumor tissues from the A549 cell line transfected with WT KEAP1, the expression levels of NRF2 and its target protein $\mathrm{HO}-1$ were significantly increased in the tumor tissues from the A549 cell line transfected with the R320Q KEAP1 mutant (Fig. 3d). The mRNA levels of NRF2 and its target genes HMOX1, HO-1, GCLC, and NQO1 in the grafted tumor tissues from the A549 cell line transfected with the R320Q KEAP1 mutant were remarkably increased compared with that in grafted tumor tissues from the A549 cell line transfected with WT KEAP1(Fig. 3c).

\section{The NRF2 inhibitor ML385 inhibited proliferation of lung cancer cells carrying KEAP1 mutations}

Increased cellular oxidative stress levels by smallmolecule compounds to enhance cytotoxicity have been identified as a viable cancer treatment strategy [26]. High levels of ROS not only inhibit cancer cell proliferation but also trigger apoptosis. A subset of NRF2 inhibitors has been reported to inhibit the proliferation of cancer cells by down-regulating the expression of NRF2, resulting in elevated levels of intracellular ROS and increased cytotoxicity. However, it is unknown whether NRF2 inhibitors have different effects on these LSCCs with or without KEAP1 somatic mutations. Thus, we selected an effective NRF2 inhibitor, ML385, which specifically and directly interacts with NRF2 protein, blocks NRF2 transcriptional activity, and enhances the efficacy of carboplatin and other chemotherapeutic drugs in lung cancer cells [27]. The lung cancer cell line A549 transfected with the R320Q KEAP1 mutant (KEAP1-R320Q mutant) or with WT KEAP1 (KEAP1-WT) and H1299 lung cancer cells, which carry both WT KEAP1 and NRF2, were selected and treated with ML385. The number of formed colonies was decreased in all three groups

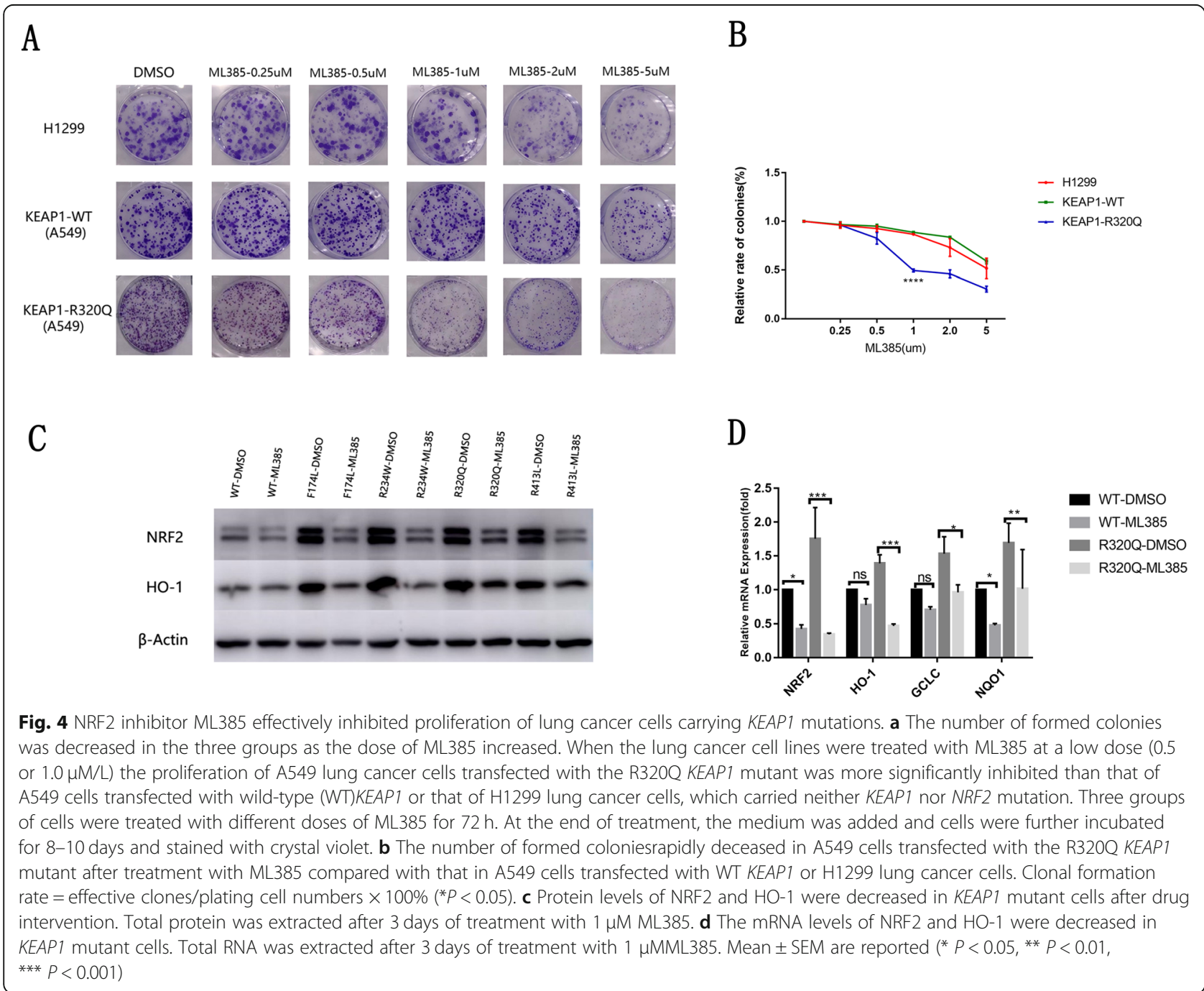


in a dose-dependent manner with increased ML385 treatment (Fig. 4a). Interestingly, when the lung cancer cell lines were treated with ML385 at a low dose, such as 0.25 or $0.5 \mu \mathrm{M} / \mathrm{L}$, the proliferation of the 549 lung cancer cell line transfected with the R320Q KEAP1 mutant showed more significant inhibition than that of A549 cells transfected with WT KEAP1 or that of H1299 lung cancer cells (Fig. 4a, b). The proliferation of lung cancer cell lines showed no significant difference between A549 cells transfected with WT KEAP1 and H1299 lung cancer cells without KEAP1 and NFR2 mutation (Fig. 4a, b). These results suggest that lung cancer cell lines with KEAP1 mutations may have higher sensitivity to ML385 treatment.

To further detect whether the effect of ML385 on lung cancer cell proliferation is mediated by inhibiting the KEAP1/NRF2 pathway, the expression of NRF2 and its target genes HO-1, GCLC and NQO1 in lung cancer cell lines treated with ML385 were investigated by western blot and real-time PCR. As shown in Fig. 4c, the expression levels of HO-1 and NRF2 protein in A549 lung cancer cells transfected with F174L, R234W, R320Q, and R413L KEAP1 mutants were significantly inhibited by ML385. However, the expression levels of HO-1 and NRF2 protein showed no significant difference between A549 transfected with WT KEAP1 treated with and without ML385 (Fig. 4c). Although mRNA expression levels of NRF2 and its target gene NQO1 in A549 cells transfected with WT KEAP1 were significantly inhibited by ML385, the mRNA expression levels of the NRF2 target genes GCLC and HO-1 were not influenced by ML385 (Fig. 4d). Notably, the mRNA expression levels of NRF2 and its target genes HO-1, GCLC, and NQO1 in A549 cells transfected with F174L, R234W, R320Q, and R413L KEAP1 mutants were dramatically decreased after treatment with ML385 (Fig. 4d).

\section{Discussion}

Recently, high-frequency somatic mutations of KEAP1 and NRF2 in the oxidative stress response pathway have been identified in patients with NSCLC by large-scale genomic studies [28]. Previous studies have revealed that NRF2 is involved in cancer development, especially lung cancer [29-32]. In addition, recent evidence suggests that in mouse models of lung cancers, activated Nrf2 inhibits the Fbxo22-dependent degradation of Bach1 via induction of Ho-1 expression, and high levels of Bach1 promoting metastasis [33]. The loss-of-function mutation of KEAP1 promoted the tumorigenesis of Kras- and Pten-driven lung cancer cell lines in mice [20, 34]. Moreover, the KEAP1/NRF2 pathway synergized with TP53 deletion mutation could induce LSCC and radiation resistance [22]. However, no evidence that KEAP1 or NRF2 mutations identified in lung cancer patients are involved in tumorgenesis has been reported. In the present study, we found that the ability of the antioxidative stress response mediated by activation of the KEAP1/NRF2 pathway is higher in lung cancer cell lines with KEAP1 mutation (A549, NCI-H460, NCI-H838) than in lung cancer cell lines without KEAP1 mutation (NCI-H1299, NCI-H292, 95D, and SPCA1). Interestingly, compared with the lung cancer cell lines A549 and H460, which carry KEAP1 mutation, after stable transfection with WT KEAP1, the mRNA levels of NRF2 and its target genes are significantly increased in A549 and H460 lung cancer cell lines transfected with KEAP1 mutants. Moreover, colony formation and migration were increased in A549 and H460 lung cancer cell lines transfected with KEAP1 mutants. Similarly, the grafted subcutaneous tumor sizes in nude mice were significantly larger in A549 cells transfected with the R320Q KEAP1 mutant than those inA549 cells transfected with WT KEAP1. These data suggest that the somatic mutations of KEAP1 identified in Chinese patients with LSCC likely promote the development of lung cancer through activation of the antioxidative stress response in the KEAP1/ NRF2 pathway.

Although genomic analysis identified some highfrequency gene mutations from LSCC, such as TP53, PI3KCA, and SOX2, no clear operationable targets for the treatment of LSCC have been found thus far [11, 12, 35]. Practically, molecular targeted therapy improves the survival of patients with lung adenocarcinoma, but no effective targeted drugs have been identifiedin clinical trials for LSCC [36]. Convenient treatment of LSCC remains to be platinum-based chemotherapy, added with immune checkpoint inhibitors that emerged recently and has brought certain benefits for the treatment of LSCC [37]. Additionally, cancer cells acquire novel nutrient dependencies to support oncogenic growth by changing metabolic pathways, Sarah E first points that dietary restriction or enzymatic depletion of asparagine can lead to suppression of Keap1 mutant tumor growth [38]. However, the overall effects of therapy for LSCC remain grim, revealing the immediate need for an effective treatment. In the present study, we found that the loss-of-function mutation inKEAP1 promotes the development of lung cancer mediated by activation of the KEAP1/NRF2 pathway. Thus, it is tempting to presume that inhibitors of the KEAP1/NRF2 pathway will likely treat Lung cancer patients carrying KEAP1/NRF2 mutations. To date, some inhibitors of the KEAP1/NRF2 pathway have been identified, such as NRF2 inhibitors that inhibit this pathway: ML385 [27], Brucea chinensis [39], clonazepa propionate [40], luteolin, all-trans retinoic acid, and flavonoid molecular compounds [41, 42]. The NRF2 inhibitor ML385 blocks activation of the pathway by inhibiting NRF2 expression. We further 
explored the effect of ML385 on the development of lung cancer cell lines with or without KEAP1 mutations. Compared with A549 lung cancer cell lines trasfected with WT KEAP1, proliferation of the A549 lung cancer cell line trasfected with the R320Q KEAP1 mutant was dramatically inhibited by ML385. These preliminary datas suggest that ML385 inhibits the proliferation of lung cancer cells with KEAP1 mutations by blocking the KEAP1/NRF2 antioxidant stress response pathway. It will provide a new option for therapy targeted to the KEAP1/NRF2 pathway in patients with LSCC carrying the KEAP1 mutation.

\section{Conclusion}

In summary, high-frequency mutation in KEAP1 has been identified in Chinese patients with LSCC. The somatic nonsynonymous mutations in KEAP1 derived from patients with lung cancer likely promote tumorigenesis via activation of the KEAP1/NRF2 antioxidant stress response pathway. Notably, NRF2 inhibitor ML385 may inhibit the proliferation of tumor cells with KEAP1 mutation by enhancing the oxidative stress level of lung cancer cells.

\section{Supplementary information}

Supplementary information accompanies this paper at https://doi.org/10. 1186/s12964-020-00568-z.

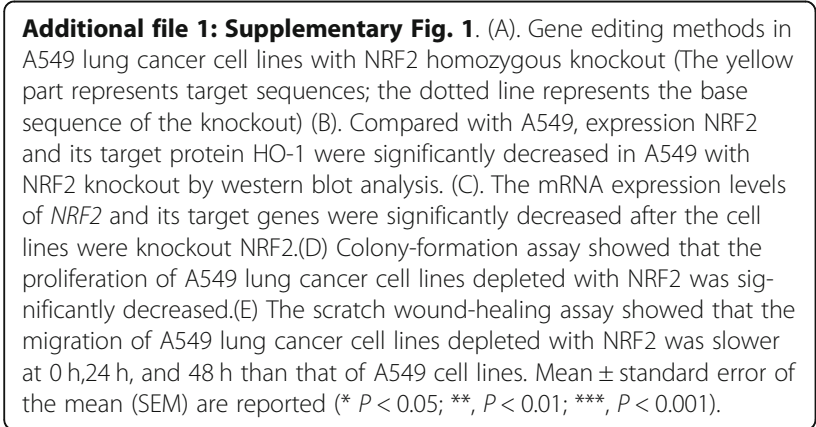

\section{Abbreviations}

NRF2/ NFE2L2: A nuclear factor erythroid 2-related factor 2; NSCLC: Nonsmallcell lung cancer; LSCC: Lung squamous cell carcinoma; EGFR: Epidermal growth factor receptor; WT: Wild-type; Polyhen2_HDI: http://genetics.bwh. harvard.edu/pph2/; SIFT: http://sift.jcvi.org/; ROS: Reactive oxygen species

\section{Acknowledgements}

Not applicable.

\section{Authors' contributions}

ML-G, Y-L and LL-Z were major contributor in writing the manuscript. CX-Z was Corresponding Author. The authors read and approved the final manuscript.

\section{Funding}

This work was supported by grants from the National Natural ScienceFoundation of China (No. 81772460 and 81472177).

Availability of data and materials Not applicable.
Ethics approval and consent to participate

Experiments with Nude mouse tumor formation experiment were carried out under the ethical approval of Research Ethics Committee of the

Shanghai Ninth People's Hospital Central Laboratory Animal Law.

\section{Consent for publication}

Not applicable.

\section{Competing interests}

The authors declare that they have no competing interests.

\section{Author details}

${ }^{1}$ Department of Respiratory and Critical Care Medicine, Yangpu Hospital, Tongji Universtiy School Of Medicine, Shanghai, China. ${ }^{2}$ Shanghai Jiaotong University School of Medicine, Shanghai, China. ${ }^{3}$ Shanghai Ninth People's Hospital, Shanghai Jiaotong University, Shanghai, China. ${ }^{4}$ Shanghai University of Traditional Chinese Medicine, Shanghai, China.

Received: 29 November 2019 Accepted: 25 March 2020

Published online: 23 June 2020

\section{References}

1. Siegel RL, Miller KD, Jemal A. Cancer statistics, 2017[J]. CA Cancer J Clin. 2017;67(1):7-30. https://doi.org/10.3322/caac.21387.

2. Chen Z, Fillmore CM, Hammerman PS, et al. Non-small-cell lung cancers: a heterogeneous set of diseases [J]. Nat Rev Cancer. 2014;14(8):535-46. https://doi.org/10.1038/nrc3775.

3. Jemal A, Siegel R, Ward E, et al. Cancer statistics, 2008[J]. CA Cancer J Clin. 2008;58(2):71-96. https://doi.org/10.3322/ca.2007.0010.

4. Fitzmaurice C, Dicker D, Pain A, et al. The global burden of cancer 2013[J]. JAMA Oncol. 2015;1(4):505-27. https://doi.org/10.1001/jamaoncol.2015.0735.

5. Paez JG, Janne PA, Lee JC, et al. EGFR mutations in lung cancer: correlation with clinical response to gefitinib therapy [J]. Science. 2004;304(5676):1497500. https://doi.org/10.1126/science.1099314.

6. Lynch TJ, Bell DW, Sordella R, et al. Activating mutations in the epidermal growth factor receptor underlying responsiveness of non-small-cell lung cancer to gefitinib [J]. N Engl J Med. 2004;350(21):2129-39. https://doi.org/ 10.1056/NEJMoa040938.

7. Felip E, Gridelli C, Baas P, et al. Metastatic non-small-cell lung cancer: consensus on pathology and molecular tests, first-line, second-line, and third-line therapy: 1st ESMO consensus conference in lung cancer; Lugano 2010[J]. Ann Oncol. 2011;22(7):1507-19. https://doi.org/10.1093/annonc/ mdr150.

8. Ju YS, Lee WC, Shin JY, et al. A transforming KIF5B and RET gene fusion in lung adenocarcinoma revealed from whole-genome and transcriptome sequencing [J]. Genome Res. 2012;22(3):436-45. https://doi.org/10.1101/gr. 133645.111.

9. Rekhtman N, Paik PK, Arcila ME, et al. Clarifying the spectrum of driver oncogene mutations in biomarker-verified squamous carcinoma of lung: lack of EGFR/KRAS and presence of PIK3CA/AKT1 mutations [J]. Clin Cancer Res. 2012;18(4):1167-76. https://doi.org/10.1158/1078-0432.ccr-11-2109.

10. Li C, Gao Z, Li F, et al. Whole exome sequencing identifies frequent somatic mutations in cell-cell adhesion genes in Chinese patients with lung squamous cell carcinoma [J]. Sci Rep. 2015;5:14237. https://doi.org/10.1038/ srep14237.

11. Kim Y, Hammerman PS, Kim J, et al. Integrative and comparative genomic analysis of lung squamous cell carcinomas in east Asian patients [J]. J Clin Oncol. 2014:32(2):121-8. https://doi.org/10.1200/jco.2013.50.8556.

12. Comprehensive genomic characterization of squamous cell lung cancers [J]. Nature. 2012:489(7417):519-25. https://doi.org/10.1038/nature11404.

13. Takahashi T, Sonobe M, Menju T, et al. Mutations in Keap1 are a potential prognostic factor in resected non-small cell lung cancer [J]. J Surg Oncol. 2010;101(6):500-6. https://doi.org/10.1002/jso.21520.

14. Kobayashi A, Kang Ml, Okawa H, et al. Oxidative stress sensor Keap1 functions as an adaptor for Cul3-based E3 ligase to regulate proteasomal degradation of Nrf2[J]. Mol Cell Biol. 2004;24(16):7130-9. https://doi.org/10. 1128/mcb.24.16.7130-7139.2004.

15. Sporn MB, Liby KT. NRF2 and cancer: the good, the bad and the importance of context [J]. Nat Rev Cancer. 2012;12(8):564-71. https://doi.org/10.1038/ nrc3278. 
16. Rotblat B, Melino G, Knight RA. NRF2 and p53: Januses in cancer?[J]. Oncotarget. 2012;3(11):1272-83. https://doi.org/10.18632/oncotarget.754.

17. Shibata T, Ohta T, Tong Kl, et al. Cancer related mutations in NRF2 impair its recognition by Keap1-Cul3 E3 ligase and promote malignancy [J]. Proc Natl Acad Sci U S A. 2008;105(36):13568-73. https://doi.org/10.1073/pnas. 0806268105.

18. Hayes JD, Dinkova-Kostova AT. The Nrf2 regulatory network provides an interface between redox and intermediary metabolism [J]. Trends Biochem Sci. 2014;39(4):199-218. https://doi.org/10.1016/j.tibs.2014.02.002.

19. Singh A, Boldin-Adamsky S, Thimmulappa RK, et al. RNAi-mediated silencing of nuclear factor erythroid-2-related factor 2 gene expression in non-small cell lung cancer inhibits tumor growth and increases efficacy of chemotherapy [J]. Cancer Res. 2008;68(19):7975-84. https://doi.org/10.1158/ 0008-5472.can-08-1401.

20. Romero R, Sayin VI, Davidson SM, et al. Keap1 loss promotes Kras-driven lung cancer and results in dependence on glutaminolysis [J]. Nat Med. 2017:23(11):1362-8. https://doi.org/10.1038/nm.4407.

21. Singh A, Bodas M, Wakabayashi N, et al. Gain of Nrf2 function in non-smallcell lung cancer cells confers radioresistance [J]. Antioxid Redox Signal. 2010;13(11):1627-37. https://doi.org/10.1089/ars.2010.3219.

22. Jeong $Y$, Hoang NT, Lovejoy A, et al. Role of KEAP1/NRF2 and TP53 mutations in lung squamous cell carcinoma development and radiation resistance [J]. Cancer Discov. 2017;7(1):86-101. https://doi.org/10.1158/21598290.cd-16-0127.

23. Adams J, Kelso R, Cooley L. The kelch repeat superfamily of proteins: propellers of cell function [J]. Trends Cell Biol. 2000;10(1):17-24.

24. Itoh K, Wakabayashi N, Katoh Y, et al. Keap1 represses nuclear activation of antioxidant responsive elements by Nrf2 through binding to the aminoterminal Neh2 domain [J]. Genes Dev. 1999;13(1):76-86.

25. Wakabayashi N, Dinkova-Kostova AT, Holtzclaw WD, et al. Protection against electrophile and oxidant stress by induction of the phase 2 response: fate of cysteines of the Keap1 sensor modified by inducers [J]. Proc Natl Acad Sci U S A. 2004;101(7):2040-5. https://doi.org/10.1073/pnas.0307301101.

26. Gorrini C, Harris IS, Mak TW. Modulation of oxidative stress as an anticancer strategy [J]. Nat Rev Drug Discov. 2013;12(12):931-47. https://doi.org/10. 1038/nrd4002.

27. Singh A, Venkannagari S, Oh KH, et al. Small molecule inhibitor of NRF2 selectively intervenes therapeutic resistance in KEAP1-deficient NSCLC tumors [J]. ACS Chem Biol. 2016;11(11):3214-25. https://doi.org/10.1021/ acschembio.6b00651.

28. Bailey $\mathrm{MH}$, Tokheim C, Porta-Pardo $\mathrm{E}$, et al. Comprehensive characterization of cancer driver genes and mutations [J]. Cell. 2018;173(2):371-385.e318. https://doi.org/10.1016/j.cell.2018.02.060.

29. Hayes JD, McMahon M, Chowdhry S, et al. Cancer chemoprevention mechanisms mediated through the Keap1-Nrf2 pathway [J]. Antioxid Redox Signal. 2010;13(11):1713-48. https://doi.org/10.1089/ars.2010.3221.

30. McCarthy N. Tumorigenesis: oncogene detox programme [J]. Nat Rev Cancer. 2011;11(9):622-3. https://doi.org/10.1038/nrc3119.

31. Hu R, Saw $C L$, Yu R, et al. Regulation of NF-E2-related factor 2 signaling for cancer chemoprevention: antioxidant coupled with antiinflammatory [J] Antioxid Redox Signal. 2010;13(11):1679-98. https://doi.org/10.1089/ars.2010. 3276.

32. Perera RM, Bardeesy N. Cancer: when antioxidants are bad [J]. Nature. 2011; 475(7354):43-4. https://doi.org/10.1038/475043a.

33. Lignitto $\mathrm{L}$, LeBoeuf $\mathrm{SE}$, Homer $\mathrm{H}$, et al. Nrf2 activation promotes lung cancer metastasis by inhibiting the degradation of Bach1[J]. Cell. 2019;178(2):316329.e318. https://doi.org/10.1016/j.cell.2019.06.003.

34. Best SA, De Souza DP, Kersbergen A, et al. Synergy between the KEAP1/ NRF2 and PI3K pathways drives non-small-cell lung cancer with an altered immune microenvironment [J]. Cell Metab. 2018;27(4):935943.e934. https://doi.org/10.1016/j.cmet.2018.02.006.

35. Fukazawa T, Guo M, Ishida N, et al. SOX2 suppresses CDKN1A to sustain growth of lung squamous cell carcinoma [J]. Sci Rep. 2016;6:20113. https://doi.org/10.1038/srep20113.

36. Perez-Moreno P, Brambilla E, Thomas $R$, et al. Squamous cell carcinoma of the lung: molecular subtypes and therapeutic opportunities [J]. Clin Cancer Res. 2012;18(9):2443-51. https://doi.org/10.1158/1078-0432.ccr-11-2370.

37. Brahmer J, Reckamp KL, Baas $P$, et al. Nivolumab versus docetaxel in advanced squamous-cell non-small-cell lung cancer [J]. N Engl J Med. 2015; 373(2):123-35. https://doi.org/10.1056/NEJMoa1504627.
38. LeBoeuf SE, Wu WL, Karakousi TR, et al. Activation of oxidative stress response in cancer generates a Druggable dependency on exogenous nonessential amino acids [J]. Cell Metab. 2020;31(2):339-350.e334. https://doi.org/10.1016/j.cmet.2019.11.012

39. Ren D, Villeneuve NF, Jiang T, et al. Brusatol enhances the efficacy of chemotherapy by inhibiting the Nrf2-mediated defense mechanism [J]. Proc Natl Acad Sci U S A. 2011;108(4):1433-8. https://doi.org/10.1073/pnas. 1014275108.

40. Choi EJ, Jung BJ, Lee SH, et al. A clinical drug library screen identifies clobetasol propionate as an NRF2 inhibitor with potential therapeutic efficacy in KEAP1 mutant lung cancer [J]. Oncogene. 2017;36(37):5285-95. https://doi.org/10.1038/onc.2017.153.

41. Lu MC, Ji JA, Jiang ZY, et al. The Keap1-Nrf2-ARE pathway as a potential preventive and therapeutic target: an update [J]. Med Res Rev. 2016;36(5): 924-63. https://doi.org/10.1002/med.21396.

42. Menegon S, Columbano A, Giordano S. The dual roles of NRF2 in cancer [J]. Trends Mol Med. 2016;22(7):578-93. https://doi.org/10.1016/j.molmed.2016. 05.002

\section{Publisher's Note}

Springer Nature remains neutral with regard to jurisdictional claims in published maps and institutional affiliations.
Ready to submit your research? Choose BMC and benefit from:

- fast, convenient online submission

- thorough peer review by experienced researchers in your field

- rapid publication on acceptance

- support for research data, including large and complex data types

- gold Open Access which fosters wider collaboration and increased citations

- maximum visibility for your research: over $100 \mathrm{M}$ website views per year

At BMC, research is always in progress.

Learn more biomedcentral.com/submissions 